\title{
Comment on self-consistent model of black hole formation and evaporation
}

\author{
Pei-Ming Ho \\ Department of Physics and Center for Theoretical Sciences, \\ Center for Advanced Study in Theoretical Sciences, \\ National Taiwan University, Taipei 106, Taiwan, R.O.C. \\ E-mail: pmho@phys.ntu.edu.tw
}

ABSTRACT: In an earlier work, Kawai et al. proposed a model of black-hole formation and evaporation, in which the geometry of a collapsing shell of null dust is studied, including consistently the back reaction of its Hawking radiation. In this note, we illuminate the implications of their work, focusing on the resolution of the information loss paradox and the problem of the firewall.

KeYwords: Black Holes, Classical Theories of Gravity

ArXIV EPRINT: 1505.02468 


\section{Contents}

1 Introduction 1

2 Geometry outside collapsing shell $\quad 3$

3 Evaporation 5

4 Proof of no horizon $\quad 6$

$\begin{array}{lll}5 & \text { Geometry of collapsing shell } & 7\end{array}$

6 Geometry of full space-time $\quad 9$

$\begin{array}{lll}7 & \text { Infalling observer } & 10\end{array}$

8 Information and firewall $\quad 11$

9 Generalization $\quad 12$

$\begin{array}{ll}10 \text { Summary } & 13\end{array}$

\section{Introduction}

The information loss paradox [1] has intrigued theoretical physicists since Hawking's discovery that black holes evaporate. The unitarity of quantum mechanics demands that Hawking radiation be not thermal, and that it carry information of the matter inside the horizon. However, to propose a mechanism to transfer information from the collapsed matter to Hawking radiation, there are various theoretical obstacles such as the no-hair theorem, the no-cloning theorem, the monogamy of entanglement, as well as the causality and locality of semiclassical effective theories. An important progress was the proof of the small-correction theorem [2], which states that the information loss cannot be recovered by anything less than order-one correction at the horizon. See ref. [2] for a more precise explanation of the information loss paradox.

As Hawking radiation is originated from the horizon, the collapsing matter has to leave all of its information before entering the horizon (unless the information can travel non-locally from the inside to the outside of the horizon at a later time). On the other hand, it is commonly believed that nothing special should happen to an infalling observer at the horizon, and so the information should be carried into the horizon. The black-hole complementarity $[3,4]$ is thus proposed as a resolution of the puzzle.

The puzzle can be presented through the Penrose diagram for the conventional model of the formation and evaporation of a black hole. (See figure 1.) An object falling in through 


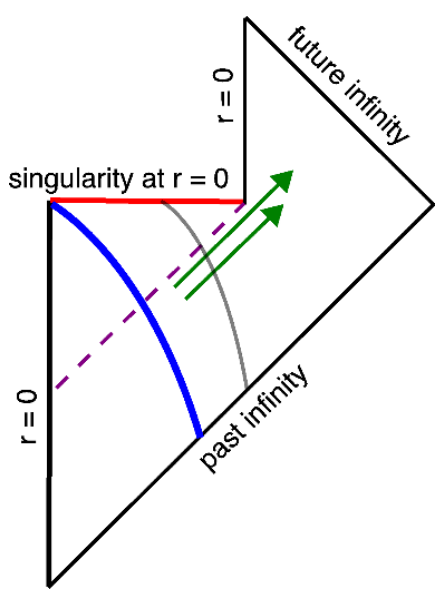

Figure 1. Penrose diagram for the conventional model of the formation and evaporation of a black hole: the blue curve represents the collapsing matter, the green arrows Hawking radiation, and the red line the singularity at the origin. The horizon is represented by the dash line, and an infalling observer the gray curve.

the horizon ends up at the singularity at $r=0$, while all of its information must come out of the horizon through Hawking radiation to a distant observer to avoid information loss. The puzzle about information arises essentially because there is a region (the region behind the horizon) from which outgoing light cones can not reach to the future infinity.

On the other hand, a different model of the (non-)formation and evaporation of a black hole was presented in the work of Kawai, Matsuo and Yokokura [5], which can be directly taken as a resolution of the information loss paradox. We will refer to their model [5] as the KMY model. It is the purpose of this article to illuminate the implications of their work on problems about the information loss paradox.

The key message of the KMY model is that the back reaction of Hawking radiation must be taken into consideration before the black hole forms. ${ }^{1}$ The formation and evaporation of a black hole happen at the same time as a single process. Hawking radiation appears before there is a horizon, and as it takes away energy from the system, its back reaction makes it harder for the horizon to emerge. In fact, it was shown [5] that no horizon ever appears (unless it is already there in the initial state), not only from the viewpoint of a distant observer, but also from the viewpoint of an infalling observer. The collapsing matter never completely falls inside the Schwarzschild radius. As a result, the conventional assumption of the empty horizon of a black hole is replaced by the presence of collapsing matter around the Schwarzschild radius, and the information loss paradox is resolved. Similar viewpoints were taken in $[6,7]$, although the reasoning and arguments are different.

Since there will never be a horizon, strictly speaking there is no black hole. In the literature, a to-be-formed black hole is called an incipient black hole, which will sometimes also be simply called a black hole in this note. ${ }^{2}$

\footnotetext{
${ }^{1}$ The Hawking radiation occurring before the formation of a black hole is sometimes called a pre-Hawking radiation.

${ }^{2}$ An incipient black hole can also have a dark surface and Hawking radiation, just like a real black hole.
} 
In this note, we will point out the implication of the KMY model that there is no horizon regardless of the nature of the collapse of matter. We will give a proof of no horizon based on semiclassical calculations. We will also point out another implication of the KMY model, which reduces the firewall $[8,9]$ to a weak radiation.

Incidentally, one may wonder what happens to a static black hole. A static black hole can be constructed through an adiabatic process in a heat bath, with the heat inflow balanced by Hawking radiation, and it was found that there is still no horizon $[5,10]$. We will not discuss this case in this note.

\section{Geometry outside collapsing shell}

It is well known that, from the viewpoint of an observer outside the black hole, the motion of an infalling particle gets slower as it gets closer to the horizon, and it can never cross the horizon. Due to Hawking radiation, the black hole evaporates and disappears within a finite amount of time. Therefore, for a distant observer, the black hole evaporates before the infalling particle reaches the horizon. This does not however imply that an infalling object cannot cross the horizon within finite proper time. According to the Penrose diagram of the traditional view of the black-hole space-time (figure 1), an infalling object can pass through the horizon in finite proper time. But to a distant observer it never reaches the horizon until the horizon shrinks to zero so that it touches the singularity at the origin at the same moment when the black hole is completely evaporated.

The KMY model claims, however, that if the effect of the back reaction of Hawking radiation is included from the very beginning, the Penrose diagram has to be significantly modified. An infalling observer can never pass the horizon, as there is no horizon.

The KMY model describes the collapse of a spherical matter shell, and the corresponding space-time geometry. Both the collapsing shell and the Hawking radiation are approximated by spherical configurations. It also ignores massive particles in the Hawking radiation. But it includes the back-reaction of Hawking radiation on the geometry, and thus the time-dependence of the Schwarzschild radius.

In this section, we study the space-time geometry outside the shell in the semiclassical approximation. The energy-momentum tensor in the Einstein equation

$$
G_{\mu \nu}=8 \pi\left\langle T_{\mu \nu}\right\rangle
$$

is given by the expectation value of the quantum energy-momentum operator of matter fields, which is identified with the Hawking radiation outside the collapsing shell. It is assumed that there is nothing but Hawking radiation outside the spherical matter shell.

Under these assumptions, the most general solution to Einstein's equation for the outside of a collapsing spherical shell is the outgoing Vaidya metric [11]: ${ }^{3}$

$$
d s^{2}=-\left(1-\frac{a(u)}{r}\right) d u^{2}-2 d u d r+r^{2} d \Omega^{2} .
$$

\footnotetext{
${ }^{3}$ Both the ingoing and outgoing Vaidya metrics have been applied to the problem of black-hole formation and evaporation in the literature. See, e.g. [12, 13].
} 
In the units $G=c=1$, the Bondi mass for this metric is $M(u)=a(u) / 2$ and the only non-vanishing component of the Einstein tensor is

$$
G_{u u}=-\frac{\dot{a}(u)}{r^{2}} .
$$

This corresponds to an energy-momentum tensor with the only non-vanishing component

$$
T_{u u}=\frac{1}{8 \pi} G_{u u}=-\frac{1}{8 \pi} \frac{\dot{a}(u)}{r^{2}} .
$$

The weak energy condition demands that $\dot{a}<0$.

The outgoing Vaidya metric has been known as a solution to Einstein's equation for a spherical star emitting null dust. Referring to the outer radius of the collapsing shell as $R(u)$, the metric (2.2) should be valid for the outside of a spherical collapsing shell for all $r \geq R(u)$, assuming that there are only massless particles in Hawking radiation [5].

Recall that the geometry outside a static mass at the origin is given by the Schwarzschild solution:

$$
d s^{2}=-\left(1-\frac{a_{0}}{r}\right) d t^{2}+\left(1-\frac{a_{0}}{r}\right)^{-1} d r^{2}+r^{2} d \Omega^{2},
$$

where the Schwarzschild radius is

$$
a_{0}=2 M,
$$

and $M$ is the total mass. It can also be rewritten in terms of the outgoing EddingtonFinkelstein coordinates as

$$
d s^{2}=-\left(1-\frac{a_{0}}{r}\right) d u^{2}-2 d u d r+r^{2} d \Omega^{2}
$$

where

$$
u=t-r^{*}(r), \quad r^{*}(r) \equiv r+a_{0} \log \left|\frac{r}{a_{0}}-1\right|,
$$

through a change of coordinates. This metric is in the form of the outgoing Vaidya metric (2.2), and we will refer to $a(u)$ in (2.2) as the Schwarzschild radius. As energy is radiated away through Hawking radiation, the Bondi mass $M(u)$ decreases with time, and so is the Schwarzschild radius $a(u)$.

Let us emphasize here that, despite the connection between the outgoing Vaidya solution and the Schwarzschild solution, whether there is a horizon for the outgoing Vaidya metric (2.2) is still a question to be answered. The initial state of the collapsing shell is assumed to satisfy $R\left(u_{1}\right)>a\left(u_{1}\right)$ at the initial time $u=u_{1}$. Since the shell continues collapsing, and the energy flux in Hawking radiation is tiny for a large initial mass $M\left(u_{1}\right)$, it is natural to expect that at some later point a horizon would appear when the shell shrinks to a size smaller than the Schwarzschild radius. Our task is to examine carefully whether this naive expectation is really what Einstein's equation tells us through the outgoing Vaidya metric.

If there really would be a horizon, at least one of the two following things must happen: (i) $R(u)<a(u)$ at a later time $u>u_{1} ;^{4}$ or (ii) the outgoing Vaidya metric (2.2) is

\footnotetext{
${ }^{4}$ We do not have to assume that $r=a(u)$ is the horizon. But if the horizon exists, $R(u)$ will go to zero after passing through the horizon, so that $R(u)$ should not have a lower bound at any finite value such as $a(u)$.
} 
geodesically incomplete, so that a horizon can hide behind the infinity $u=\infty$. We will show in the following that neither of the two things happen, so there is no horizon, contrary to the naive expectation.

\section{Evaporation}

One may wonder if Hawking radiation would still appear if there is no horizon. We argue that, as the collapsing shell gets very close to the Schwarzschild radius, the geometry just outside the shell is indistinguishable from the near horizon region of a real black hole and thus Hawking radiation is expected to appear, although its spectrum can be modified. This view is supported by the literature [14-19].

In fact, it has been calculated in this context in [5], in the absence of a horizon. Hawking radiation appears on the shell as well as outside the shell. The energy flux in Hawking radiation determines the rate of change in the mass $\dot{M}(u)=\dot{a}(u) / 2$. In 4 dimensions, the Hawking temperature $T_{H}(u)$ is roughly $1 / a(u),{ }^{5}$ and the energy flux in the radiation of massless particles is [5]

$$
J \propto \text { Area } \times T_{H}^{4} \propto \frac{1}{a^{2}(u)} .
$$

Thus in the semiclassical, spherical symmetry approximation,

$$
\dot{a}(u)=-C / a^{2}(u)
$$

for some constant $C . \quad\left(C \equiv \frac{N}{48 \pi}\right.$, which is proportional to the number $N$ of species of massless particles.) The solution is

$$
a(u)= \begin{cases}3 C^{1 / 3}\left(u_{0}-u\right)^{1 / 3} & \left(u<u_{0}\right) \\ 0 & \left(u \geq u_{0}\right)\end{cases}
$$

for some constant $u_{0}$ when the matter shell is completely evaporated. The only information we need from this calculation is that the Schwarzschild radius $a(u)$ decreases monotonically to zero in a finite elapse in $u$, and after that the spacetime becomes Minkowskian. The argument below for the absence of horizon will not rely on the detail of this solution (3.3).

Of course, the semiclassical analysis of Hawking radiation is not expected to be valid all the way to the Planck scale. In this work we are not really concerned with the fate of the collapsing shell when it is reduced to a Planck size. Our aim is to understand the semiclassical physics involved to explain the formation and evaporation of an astronomical black hole. We will say that a macroscopic black hole is completely evaporated even when there are Planck-scale remnants (see e.g. [20]), whose entropy is negligible compared with an astronomical massive object. In this sense we assume that an incipient black hole evaporates completely within finite $u$.

Notice that it is generally assumed that a macroscopic black hole evaporates away in finite time $t$. At large $r(r \gg a(u))$, we have $d t \simeq d u+d r$ for the proper time $t$ of a distant observer located at a fixed $r$, hence an elapse in finite $t$ means the same as finite $u$.

\footnotetext{
${ }^{5}$ This is an approximation of a more precise expression [5] for large $a$.
} 


\section{Proof of no horizon}

To prove that there is no horizon, we will show the following two things: (i) $R(u)>a(u)$ for all $u>u_{1}$ as long as $R\left(u_{1}\right)>a\left(u_{1}\right)$ at an initial time $u=u_{1}$, until the collapsing shell is completely evaporated; and (ii) when the collapsing shell is completely evaporated, $R(u)>0$ and extends into the Minkowski space, which is geodesically complete, so that a horizon cannot hide behind the infinity $u=\infty$.

First we sketch a proof by contradiction to prove that the outer radius of the collapsing shell is always outside the Schwarzschild radius, in the context of semiclassical theories with minimal assumptions.

Assume that $R(u)$ eventually falls inside $a(u)$. The outer radius would have to pass through the Schwarzschild radius, with the velocity of the outer surface $(r=R(u))$ larger (moving faster towards the origin) than the velocity of the Schwarzschild radius $(r=a(u)$ ) at a certain instant $u=u^{\prime}$ when $R\left(u^{\prime}\right)=a\left(u^{\prime}\right)$. The outgoing Vaidya metric (2.2) would be applicable to the point $r=a(u)$ for $u \geq u^{\prime}$, and the trajectory of the Schwarzschild radius $(r=a(u))$ would be space-like: ${ }^{6}$

$$
d s^{2}=-2 d u d a(u)=-2 d u^{2} \dot{a}(u)>0 \quad\left(u \geq u^{\prime}\right)
$$

because $\dot{a}<0$. As the trajectory of a point on the outer surface of the shell $(r=R(u))$ is either light-like or time-like, it is impossible for the outer surface of the shell to move faster towards the origin than the Schwarzschild radius, and thus we have a contradiction.

In other words, if $R(u)>a(u)$ for an initial state at $u=u_{1}$, it is impossible to have $R(u)<a(u)$ at a later time $u>u_{1}$. The radius $R(u)$ of the collapsing shell can never catch up with the Schwarzschild radius $a(u)$, and the horizon can never emerge, at least when the outgoing Vaidya metric (2.2) is still valid.

The outgoing Vaidya metric remains valid as it turns into the Minkowski space metric when $a(u)=0$ after the shell disappears at $u=u_{0}$. In figure 2 , we demonstrate the trajectories of $R(u)$ for different initial conditions, assuming that the outer radius of the matter shell shrinks at the highest speed possible - the speed of light, which is still slower than $a(u)$. Hence, for arbitrary initial conditions, $R\left(u_{0}\right)>0$ and it extends into the Minkowski space smoothly. As the Minkowski space has no horizon and is geodesically complete, the trajectory of $R(u)$ is geodesically complete without crossing the Schwarzschild radius anywhere. At a speed lower than or equal to light, an infalling observer originally outside the collapsing shell would therefore also have a geodesically complete trajectory without crossing a horizon (or hitting any singularity). We conclude that there can be no horizon at all for a collapsing matter shell no matter how fast it collapses.

A few assumptions were made in the argument above. The use of the Vaidya metric has to do with the fact that the emission of massive particles in Hawking radiation is ignored. But it is a robust feature that the trajectory $r=a$ of the Schwarzschild radius is space-like when $\dot{a}<0$. This is because the trajectory $r=a_{0}$ is null-like when $a=a_{0}$

\footnotetext{
${ }^{6}$ Let us emphasize that we are showing a proof by contradiction. We are not saying that the trajectory $r=a(u)$ is actually space-like, as the outgoing Vaidya metric is not applicable to it with $R(u)>a(u)$. In the next section, we will show more directly that $R(u)>a(u)$ at all times.
} 


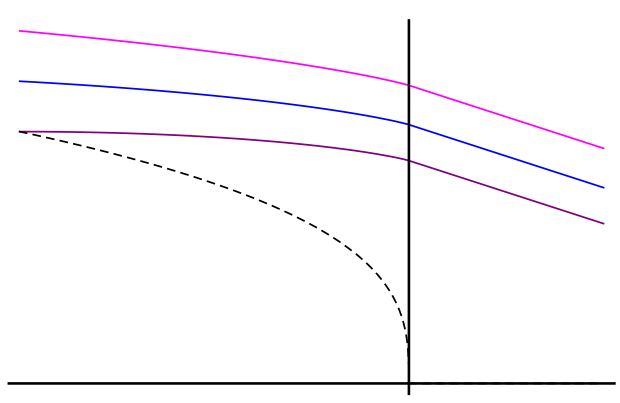

(a)

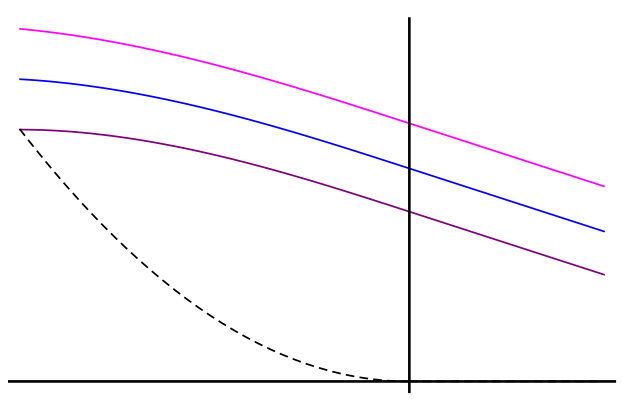

(b)

Figure 2. In both figures, the horizontal axis is $u$, and the vertical axis for $r$ is located at $u=u_{0}$, where the matter shell is completely evaporated, and the spacetime is Minkowskian for $u \geq u_{0}$. The dashed curve represents $a(u)$, and the solid curves $R(u)$ for different initial conditions. The two figures differ in the trajectory of $a(u)$. It is $a(u) \propto\left(u_{0}-u\right)^{1 / 3}$ in (a) and $a(u) \propto\left(u_{0}-u\right)^{2}$ in (b). It is a robust feature that $R(u)$ remains at a finite value at $u_{0}$ when $a(u)$ goes to zero.

is constant. If we had used the metric $(2.5)$ and replaced $a_{0}$ by $a(t)$ with $\dot{a}(t)<0$, we could still show that, for any finite $|\dot{a}(t)| \neq 0$, the trajectory $r=a(t)+\epsilon$ of a point just outside the Schwarzschild radius is space-like for sufficiently small $\epsilon>0$. Furthermore, the inclusion of massive particles in Hawking radiation would lead to a faster evaporation, making it even harder for $R(u)$ to catch up with $a(u)$.

In addition to spherical symmetry, we have also assumed that $\dot{a}<0$ due to Hawking radiation until $a\left(u_{0}\right)=0$, and that the shell evaporates completely at a finite value of $u=u_{0}$. The value of $|\dot{a}|$ can otherwise be arbitrarily small.

Note that we have not made any assumption about the constituents of the matter shell, except that its trajectory is time-like or light-like, unlike other works with a similar conclusion $[21,22]$.

\section{Geometry of collapsing shell}

Let us give more details for the simple example of a collapsing matter shell composed of massless dust [5]. The special case of null dust is interesting because if even a shell collapsing at the speed of light cannot form a horizon, a generic time-like collapsing shell most certainly cannot, either.

For spherically symmetric configurations, the light-cone directions for the outgoing Vaidya metric (2.2) are given by

$$
d u=0, \quad\left(1-\frac{a(u)}{r}\right) d u+2 d r=0 .
$$

The solutions to the first equation $d u=0$ give trajectories of the outgoing massless particles in Hawking radiation. The solutions to the second equation then describe the trajectories of infalling massless particles, including those at the outer surface of the shell of null dust. 


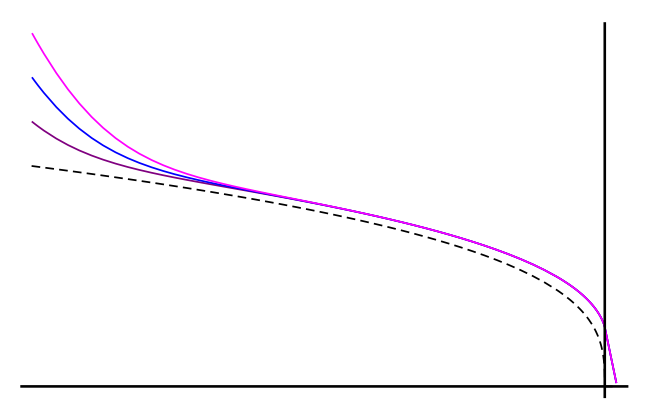

Figure 3. The horizontal axis is $u$ and the vertical axis is located at $u=u_{0}$, where $a\left(u_{0}\right)=0$. The trajectories of $R(u)$ as solutions of (5.2) for different initial conditions are given, in comparison with that of $a(u)$ given by $(3.3)$ as a dashed curve. For each trajectory of $R(u)$, it starts from its initial value on the left and approaches $a(u)$ quickly. The middle part is described by (5.3) when $R(u)$ is very close to $a(u)$. When $a(u)$ goes to $0, R(u)$ is approximated by $(5.4)$, and this regime was the focus of figure $2(\mathrm{a})$, although with different initial conditions.

Therefore, the outer radius $R(u)$ of the collapsing spherical shell satisfies [5]

$$
\frac{d R(u)}{d u}=-\frac{1}{2}\left(1-\frac{a(u)}{R(u)}\right)
$$

According to this equation, $d R / d u \rightarrow 0$ as $R \rightarrow a$, and so $R$ can never get inside the Schwarzschild radius.

Samples of the null-like trajectories of $R(u)$ for different initial conditions at $u_{1}<u_{0}$ are illustrated in figure 2 and figure 3. The fact that $R(u)$ remains outside the Schwarzschild radius $a(u)$ is a robust feature that does not rely on the explicit dependence of $a(u)$ on $u$, but only the fact that $\dot{a}<0$. In general, since the trajectory of $r=R(u)$ is null-like but that of $r=a(u)$ is space-like. $R(u)$ can never catch up with $a(u)$ before $u_{0}$, regardless of the initial condition. The trajectory of $R(u)$ can always be smoothly extended into the Minkowski space, and thus the trajectory of $R(u)$ is geodesically complete.

For a sufficiently large collapsing shell, typically $R(u)$ gets very close to the Schwarzschild radius for a long period of time (see figure 3). During the stage when $R(u)$ is very close to $a(u),{ }^{7}$ to the first order approximation, the solution of $R(u)$ to the equation above is [5]

$$
R(u)=a(u)-2 a(u) \dot{a}(u)+\cdots .
$$

This is a good approximation if $|\dot{a}| \ll 1$, when the outer radius of the shell gets close to the Schwarzschild radius $(|R-a| \ll a)$, before the matter shell evaporates towards the Planck scale when $a \rightarrow 0$.

At the final stage of evaporation $\left(u \sim u_{0}\right.$ but $\left.u<u_{0}\right)$, with the Schwarzschild radius $a(u) \sim 0$, the trajectory of $R(u)$ can be solved from the light-cone condition (5.2) in the limit $u \rightarrow u_{0}^{-}($when $a(u) \ll R(u))$ by

$$
R(u) \rightarrow \frac{u_{0}-u}{2}+c
$$

\footnotetext{
${ }^{7}$ Notice that we are studying the separation between $R(u)$ and $a(u)$ as values of the coordinate $r$, which is not the physical distance. This is nevertheless sufficient for our purpose to show the absence of horizon.
} 
for some constant $c>0$ determined by the initial condition. (See figure 2.) Hence the matter shell remains a finite size when it completely evaporates.

Since the trajectory of $R(u)$ is null-like and is geodesically complete, it is impossible for a time-like or null-like geodesic originated from the region outside the shell to pass through the Schwarzschild radius, as we commented in the previous section.

\section{Geometry of full space-time}

In the above we have discussed the geometry of the region outside the matter shell. The space enclosed by the collapsing shell is by assumption in vacuum (with vanishing energy momentum tensor), hence it has to be the Minkowski space, as required by Birkhoff's theorem.

To understand the geometry of the region occupied by the collapsing matter, one can divide the collapsing shell of finite thickness into infinitely many infinitesimally thin layers, separated by infinitesimal gaps [5]. For the inner most layer of dust, the space inside is Minkowskian. Assuming that the dust cannot pass through itself, the first layer comes in at the speed of light and piles up at the origin. The second layer comes in after that, slowing down at the Schwarzschild radius defined by the first layer. Then the third layer falls in, and so on. This description can also be applied to a collapsing solid sphere as well.

One can treat the trajectory of each layer in a way similar to what was done for $R(u)$ in the above [5]. For a sufficiently large and dense body of collapsing matter, eventually the radius of each layer approaches to its own Schwarzschild radius defined for the total mass enclosed by that layer, until the last stage when the evaporation is approaching the Planck scale.

The ultimate fate of the collapsing shell at the Planck scale (whether it evaporates completely) and the resolution of the singularity at the origin (if any) by a UV-complete theory are outside the scope of this article. Semiclassical considerations of the final stage of the evaporation can be found in e.g. [5, 23, 24]. The purpose of this article is to resolve puzzles about black holes at the semiclassical level as much as possible. It does not exclude the potential relevance of Planck-scale physics such as string theory to black holes (e.g. through the idea of the fuzzballs $[25,26])$ at a more detailed level.

According to our discussions above, the whole space-time can be divided into four regions:

1. the Minkowskian region inside the shell with the metric

$$
d s^{2}=-d U^{2}-2 d U d r+r^{2} d \Omega^{2} .
$$

2. the region occupied by the collapsing matter shell.

3. the region outside the shell with the outgoing Vaidya metric (2.2).

4. the Minkowskian region after the shell completely evaporates $\left(a(u)=0\right.$ for $u>u_{0}$ in the Vaidya metric). 


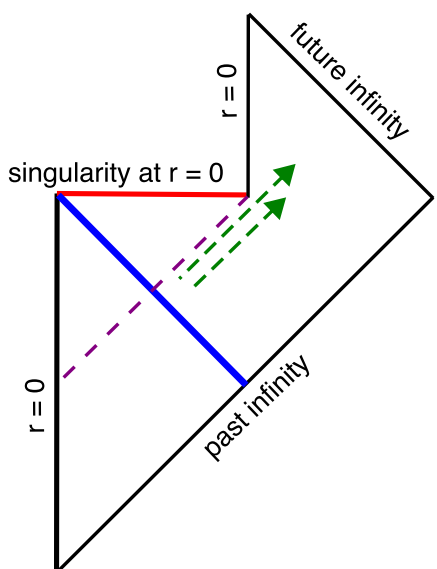

(a)

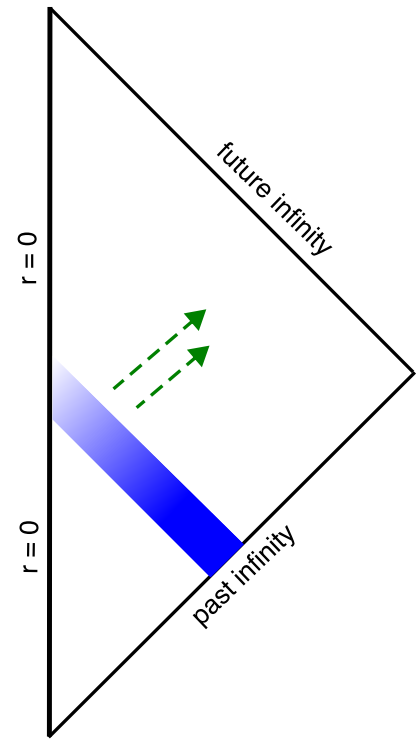

(b)

Figure 4. In both diagrams, blue lines represent collapsing null matter shells, green arrows Hawking radiation. Figure (a) shows the Penrose diagram for the conventional view of a black hole formed by a collapsing null matter shell. Figure (b) shows the Penrose diagram for the collapsing null matter shell according to the KMY model.

Put together, they constitute a geodesically complete spacetime. The corresponding Penrose diagram is depicted in figure 4(b), along with the Penrose diagram for the conventional view in figure 4(a) for comparison.

\section{$7 \quad$ Infalling observer}

In principle, since the distant observer's view is already complete, there is no need to consider the viewpoint of an infalling observer. Nevertheless, we repeat here the conventional story about how an infalling observer passes through the horizon, and point out the reason why it breaks down in the KMY model.

The (static) Schwarzschild solution can be expressed in the ingoing EddingtonFinkelstein coordinates as

$$
d s^{2}=-\left(1-\frac{a_{0}}{r}\right) d v^{2}+2 d v d r+r^{2} d \Omega^{2}
$$

where

$$
v=t+r^{*}(r) .
$$

The light-like trajectories are given by

$$
\frac{d r}{d v}=-\infty, \quad \frac{d r}{d v}=\frac{1}{2}\left(1-\frac{a_{0}}{r}\right) .
$$

An infalling trajectory with $\frac{d r}{d v}<0$ moves at a speed slower than light as long as $\frac{d r}{d v}$ is finite. 
The proper time it takes an infalling observer to reach the Schwarzschild radius from $r=a_{0}+\epsilon\left(a_{0} \gg \epsilon>0\right)$ is

$$
\int d s=\int_{a_{0}}^{a_{0}+\epsilon} d r \sqrt{\left(1-\frac{a_{0}}{r}\right)\left(\frac{d r}{d v}\right)^{-2}-2\left(\frac{d r}{d v}\right)^{-1}},
$$

which is finite as long as $\left|\frac{d r}{d v}\right|^{-1}$ is finite.

Note that $v$ is related to $u$ via

$$
v=u+2 r^{*},
$$

where $r^{*}$ is given in (2.8), so that a point on the horizon $r=a_{0}$ at finite $v$ corresponds to $u=\infty$. This is why a distant observer can never see the infalling observer to pass the horizon of a static black hole.

It is conventionally assumed that for a very small Hawking radiation, there is no reason for the infalling observer to have a dramatically different experience. The infalling observer is still expected to be able to pass through the horizon within finite proper time.

In the KMY model, the geometry outside the collapsing shell looks just like that of a black hole. The effect of a small Hawking radiation also makes a small difference to a distant observer. However, the matter shell evaporates away in finite time and the space is Minkowkian for $u>u_{0}$. A coordinate transformation of the form (7.5) implies that the infalling observer should fall into the Minkowski space before $r$ reaches the Schwarzschild radius $a(u)$, because $r \rightarrow a$ implies $r^{*} \rightarrow-\infty$, which in turn implies $u \rightarrow \infty$ for a finite $v$, and we have $a(u)=0$ for large $u$.

Therefore, in agreement with the viewpoint of the distant observer, the infalling observer can never pass through the Schwarzschild radius. From the viewpoints of both the distant observer and the infalling observer, the collapsing shell as well as the infalling observer stay outside the Schwarzschild radius at all times. They both see the collapsing shell evaporate completely, and the space-time becomes Minkowskian again after all of the Hawking radiation dissipates to the infinity.

\section{Information and firewall}

From the viewpoint of a distant observer, as a collapsing matter shell gets closer to the Schwarzschild radius, the shell gets dimmer and looks more like a black hole. While the matter never falls inside the horizon, Hawking radiation is created in the neighborhood of the matter. It is thus natural to assume that Hawking radiation carries the information about the details of the matter shell, and the information loss paradox is resolved.

It used to be a common belief that, for a large black hole, an infalling observer can pass through the horizon without feeling anything dramatically different from the ordinary Minkowski space in vacuum. We find that, on the contrary, if the effect of Hawking radiation is consistently taken into account before the horizon appears, as it is in the KMY model, the surface of the collapsing matter stays outside the Schwarzschild radius, so it is impossible for an infalling observer to mistakenly assume that the space around the Schwarzschild radius is in vacuum. 
In fact, it is proven as a theorem [2] that the unitarity of quantum mechanics is inconsistent with the assumption that nothing happens at the horizon, and fuzzballs $[25,26]$ were proposed to replace the horizon in vacuum. A more recent proposal for an eventful horizon was that of the firewall $[8,9]$. It states that, in order for the Hawking radiation to be a pure state (when the collapsing matter comes in a pure state), there has to be a high energy flux - the so-called "firewall" — near the horizon of a sufficiently old black hole.

The firewall is essentially the blue-shifted Hawking radiation generated near the horizon, before it propagates to a distance with a large red shift. For the KMY model, we can check how much energy is there in the blue-shifted Hawking radiation. The question is whether the presence of the collapsing shell, which cuts off the near horizon region at a certain distance from $a(u)$, would reduce the firewall to a cold shower.

At the outer surface of the collapsing shell, the energy-momentum tensor of the Hawking radiation is

$$
T_{++}=\frac{d u}{d x^{+}} \frac{d u}{d x^{+}} T_{u u}=-\frac{1}{8 \pi}\left(1-\frac{a(u)}{R(u)}\right)^{-1} \frac{\dot{a}(u)}{R^{2}(u)},
$$

where $d x^{+}$is the local outgoing light-cone coordinate normalized by $d s^{2}=d x^{+^{2}}$ along an outgoing null trajectory.

If the radius $R(u)$ of the shell could get arbitrarily close to the Schwarzschild radius $a(u)$, the energy-momentum tensor $T_{++}$can be arbitrarily large at the collapsing shell due to an arbitrarily large blue shift $(1-a / R)^{-1}$. However, the formula (8.1) is valid only for $r \geq R(u)$, and $R(u)$ always lags behind $a(u)$. Hence $R(u)$ may never be sufficiently close to $a(u)$ for $T_{++}$to be incredibly large. Indeed, when the shell radius $R(u)$ is very close to $a(u)$, it can be approximated by the expression (5.3), so that

$$
T_{++} \simeq \frac{1}{16 \pi} \frac{1}{a^{2}(u)}
$$

which is very small for a large mass. For a shell collapsing at a speed slower than light, the radius $R(u)$ would be even farther away from $a(u)$, and $T_{++}$at $r=R(u)$ would be even smaller. Our conclusion is thus that there is nothing that can be justified to be called a firewall.

\section{Generalization}

The basic ideas involved in the discussions above allow straightforward generalizations to higher dimensions. The outgoing Vaidya metric in D-dimensional space-time is [27]

$$
d s^{2}=-\left(1-\frac{2 M(u)}{(D-3) r^{D-3}}\right) d u^{2}-2 d u d r+r^{2} d \Omega_{D-2}^{2}
$$

where $d \Omega_{D-2}^{2}$ is the metric for a $(D-2)$-dimensional unit sphere. In all dimensions higher than 4 , this metric can be used to describe the region outside a collapsing null shell as we did above in 4 dimensions. The Schwarzschild radius $a(u)=2 M(u) /(D-3)$ has a space-like trajectory $\left(d s^{2}>0\right)$ as long as the mass $M(u)$ decreases with time, as it should due to Hawking radiation. 
The only non-vanishing component of the energy-momentum tensor is

$$
T_{u u}=-\frac{(D-2)}{8 \pi(D-3) r^{D-2}} \dot{M}(u),
$$

which can be interpreted as that of the Hawking radiation.

We expect that the basic ingredients of the arguments above for no horizon can be readily applied to black holes in higher dimensions, and to generalized Vaidya metrics [28], as well as other metrics including the effect of Hawking radiation.

\section{Summary}

The traditional view of the formation and evaporation of a black hole depicted in figure 1 is obtained as a composition of two Penrose diagrams: the Penrose diagram for the formation of a black hole without the back reaction of Hawking radiation, and that for the asymptotically flat spacetime with Hawking radiation. The formation of a black hole and its evaporation are treated separately as independent processes.

The KMY model, on the other hand, consistently includes the effect of the back reaction of Hawking radiation from the very beginning to the very end. All infalling time-like or light-like geodesics originated from the outside of the collapsing shell can be extended to be geodesically complete without encountering any horizon. The Penrose diagram for the KMY model in figure 4(b) shows no horizon and no information loss at a macroscopic scale.

Furthermore, the conventional assumption about an empty horizon is replaced by the collapsing matter outside the Schwarzschild radius. There is no firewall because the blueshift of the Hawking radiation is cut off by the radius of the collapsing shell.

For a distant observer, it takes a longer time for light to reach him or her from the collapsing star as its outer radius gets closer to the Schwarzschild radius, hence the star looks darker. Therefore the fact that there is no horizon is not in contradiction with observational evidences of phenomenological black holes.

Despite the fact that we have ignored non-spherical and massive contributions to Hawking radiation, the qualitative features presented above should be valid for a generic incipient black hole, and provide us with a comprehensive semiclassical understanding of the information loss paradox, as well as related problems such as the firewall.

\section{Acknowledgments}

The author would like to thank Hikaru Kawai for sharing his original ideas on which this work is based. He also thanks Heng-Yu Chen, Kazuo Hosomichi, Takeo Inami, Keisuke Izumi, Samir Mathur and Chih-Hung Wu for discussions. The work is supported in part by the Ministry of Science and Technology, R.O.C. and by National Taiwan University. 
Open Access. This article is distributed under the terms of the Creative Commons Attribution License (CC-BY 4.0), which permits any use, distribution and reproduction in any medium, provided the original author(s) and source are credited.

\section{References}

[1] S.W. Hawking, Breakdown of Predictability in Gravitational Collapse, Phys. Rev. D 14 (1976) 2460 [INSPIRE].

[2] S.D. Mathur, The information paradox: A pedagogical introduction, Class. Quant. Grav. 26 (2009) 224001 [arXiv: 0909.1038] [INSPIRE].

[3] L. Susskind, L. Thorlacius and J. Uglum, The stretched horizon and black hole complementarity, Phys. Rev. D 48 (1993) 3743 [hep-th/9306069] [INSPIRE].

[4] G. 't Hooft, On the Quantum Structure of a Black Hole, Nucl. Phys. B 256 (1985) 727 [INSPIRE].

[5] H. Kawai, Y. Matsuo and Y. Yokokura, A Self-consistent Model of the Black Hole Evaporation, Int. J. Mod. Phys. A 28 (2013) 1350050 [arXiv:1302.4733] [InSPIRE].

[6] C.G. Callan Jr., S.B. Giddings, J.A. Harvey and A. Strominger, Evanescent black holes, Phys. Rev. D 45 (1992) 1005 [hep-th/9111056] [INSPIRE].

[7] C. Barcelo, S. Liberati, S. Sonego and M. Visser, Fate of gravitational collapse in semiclassical gravity, Phys. Rev. D 77 (2008) 044032 [arXiv:0712.1130] [INSPIRE].

[8] A. Almheiri, D. Marolf, J. Polchinski and J. Sully, Black Holes: Complementarity or Firewalls?, JHEP 02 (2013) 062 [arXiv:1207.3123] [INSPIRE].

[9] S.L. Braunstein, S. Pirandola and K. Życzkowski, Better Late than Never: Information Retrieval from Black Holes, Phys. Rev. Lett. 110 (2013) 101301 [arXiv:0907.1190] [INSPIRE].

[10] H. Kawai and Y. Yokokura, Phenomenological Description of the Interior of the Schwarzschild Black Hole, Int. J. Mod. Phys. A 30 (2015) 1550091 [arXiv:1409.5784] [INSPIRE].

[11] P. Vaidya, The Gravitational Field of a Radiating Star, Proc. Indian Acad. Sci. A 33 (1951) 264.

[12] W.A. Hiscock, Models of Evaporating Black Holes, Phys. Rev. D 23 (1981) 2813 [inSPIRE].

[13] Y. Kuroda, Vaidya space-time as an evaporating black hole, Prog. Theor. Phys. 71 (1984) 1422 [INSPIRE].

[14] P. Hajicek, On the Origin of Hawking Radiation, Phys. Rev. D 36 (1987) 1065 [InSPIRE].

[15] M. Visser, Essential and inessential features of Hawking radiation, Int. J. Mod. Phys. D 12 (2003) 649 [hep-th/0106111] [INSPIRE].

[16] C. Barcelo, S. Liberati, S. Sonego and M. Visser, Quasi-particle creation by analogue black holes, Class. Quant. Grav. 23 (2006) 5341 [gr-qc/0604058] [inSPIRE].

[17] C. Barcelo, S. Liberati, S. Sonego and M. Visser, Hawking-like radiation does not require a trapped region, Phys. Rev. Lett. 97 (2006) 171301 [gr-qc/0607008] [INSPIRE].

[18] C. Barcelo, S. Liberati, S. Sonego and M. Visser, Minimal conditions for the existence of a Hawking-like flux, Phys. Rev. D 83 (2011) 041501 [arXiv: 1011.5593] [INSPIRE]. 
[19] C. Barcelo, S. Liberati, S. Sonego and M. Visser, Hawking-like radiation from evolving black holes and compact horizonless objects, JHEP 02 (2011) 003 [arXiv:1011.5911] [INSPIRE].

[20] P. Chen, Y.C. Ong and D.-h. Yeom, Black Hole Remnants and the Information Loss Paradox, arXiv: 1412.8366 [INSPIRE].

[21] L. Mersini-Houghton, Backreaction of Hawking Radiation on a Gravitationally Collapsing Star I: Black Holes?, arXiv: 1406.1525 [INSPIRE].

[22] L. Mersini-Houghton and H.P. Pfeiffer, Back-reaction of the Hawking radiation flux on a gravitationally collapsing star II, arXiv:1409.1837 [INSPIRE].

[23] F. Fayos and R. Torres, Local behaviour of evaporating stars and black holes around the total evaporation event, Class. Quant. Grav. 27 (2010) 125011 [INSPIRE].

[24] M. O'Loughlin, Linear mass Vaidya metric at the end of black hole evaporation, Phys. Rev. D 91 (2015) 044020 [arXiv: 1312.4702] [INSPIRE].

[25] O. Lunin and S.D. Mathur, AdS/CFT duality and the black hole information paradox, Nucl. Phys. B 623 (2002) 342 [hep-th/0109154] [INSPIRE].

[26] O. Lunin and S.D. Mathur, Statistical interpretation of Bekenstein entropy for systems with a stretched horizon, Phys. Rev. Lett. 88 (2002) 211303 [hep-th/0202072] [INSPIRE].

[27] B.R. Iyer and C.V. Vishveshwara, The Vaidya Solution in Higher Dimensions, Pramana 32 (1989) 749.

[28] A. Wang and Y. Wu, Generalized Vaidya solutions, Gen. Rel. Grav. 31 (1999) 107 [gr-qc/9803038] [INSPIRE]. 